\title{
Lattice fermions as spectral graphs
}

\author{
Jun Yumoto ${ }^{a}$ and Tatsuhiro Misumi ${ }^{b, c}$ \\ ${ }^{a}$ Department of Mathematical Science, Akita University, \\ 1-1 Tagata-Gakuen-machi, Akita 010-8502, Japan \\ ${ }^{b}$ Department of Physics, Kindai University, \\ 3-4-1 Kowakae, Higashi-osaka, Osaka 577-8502, Japan \\ ${ }^{c}$ Research and Education Center for Natural Sciences, Keio University, \\ 4-1-1 Hiyoshi, Yokohama, Kanagawa 223-8521, Japan \\ E-mail: d8521007@s.akita-u.ac.jp, misumi@phys.kindai.ac.jp
}

ABSTRACT: We study lattice fermions from the viewpoint of spectral graph theory (SGT). We find that a fermion defined on a certain lattice is identified as a spectral graph. SGT helps us investigate the number of zero eigenvalues of lattice Dirac operators even on the non-torus and non-regular lattice, leading to understanding of the number of fermion species (doublers) on lattices with arbitrary topologies. The procedure of application of SGT to lattice fermions is summarized as follows: (1) One investigates a spectral graph corresponding to a lattice fermion. (2) One obtains a matrix corresponding to the graph. (3) One finds zero eigenvalues of the matrix by use of the discrete Fourier transformation (DFT). (4) By taking an infinite-volume and continuum limits, one finds the number of species. We apply this procedure to the known lattice fermion formulations including Naive fermions, Wilson fermions and Domain-wall fermions, and reproduce the known fact on the number of species. We also apply it to the lattice fermion on the discretized fourdimensional hyperball and discuss the number of fermion species on the bulk. In the end of the paper, we discuss the application of the analysis to lattice fermions on generic lattices with arbitrary topologies, which could lead to constructing a new theorem regarding the number of species.

KEYWorDS: Lattice QCD, Other Lattice Field Theories

ArXiv EPrint: 2112.13501 


\section{Contents}

1 Introduction 1

2 Spectral graph theory $\quad 3$

3 Naive fermion $\quad 5$

3.1 Dirac matrix of naive fermion 5

$\begin{array}{lll}3.2 & \text { Graphs corresponding to naive fermion } & 7\end{array}$

3.3 Diagonalization of Dirac matrix $\mathcal{D} \quad 8$

4 Wilson fermion $\quad 9$

4.1 Dirac matrix of Wilson fermion 9

$\begin{array}{lll}4.2 \text { Graphs corresponding to Wilson fermion } & 10\end{array}$

$\begin{array}{lll}4.3 & \text { Diagonalization of Dirac matrix } \mathcal{D}_{W} & 10\end{array}$

5 Domain-wall fermion $\quad \mathbf{1 2}$

$\begin{array}{lll}5.1 & \text { Dirac matrix and mass matrix of domain-wall fermion } & 12\end{array}$

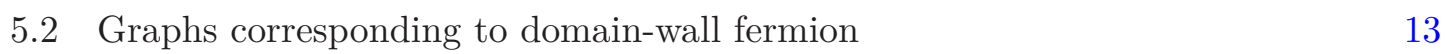

5.3 Diagonalization of Dirac matrix $\mathcal{D}_{D W}$ and mass matrix $\mathcal{M}_{D W} \quad 15$

$6 \quad$ Lattice fermions on four dimensional hyperball $\quad \mathbf{1 6}$

$\begin{array}{ll}\text { 6.1 Graphs corresponding to the Dirac matrix on hyperball } & 16\end{array}$

$\begin{array}{ll}\text { 6.2 Lattice action on four-dimensional hyperball } & 18\end{array}$

$\begin{array}{ll}6.3 & \text { Number of species on four-dimensional hyperball } \\ \end{array}$

$\begin{array}{lll}7 & \text { Summary and discussion } & 19\end{array}$

\section{Introduction}

Non-perturbative physics of quantum field theories has been investigated by lattice gauge theory $[1,2]$. Despite of the accomplishments obtained by the lattice Monte-Carlo simulation, there are still troublesome problems in lattice fermion formulations [3-6], including the reconcilement of chiral symmetry and numerical efficiency, the realization of a single Weyl fermion, and the sign problem of the quark determinant. The known lattice fermions, including Wilson, staggered and domain-wall (overlap) fermions, have their own shortcomings [7-19]. Other lattice fermion formulations have been investigated, but they also have their own drawbacks: the generalized Wilson fermions based on flavored-mass terms [20-27] and the staggered-Wilson fermions [17, 24, 28-39] can reduce numerical costs and discretizartion errors. However, their implementation to lattice QCD is complicated and eventually leads to costs. The minimally doubled fermion [40-60] with $U_{n,(1)}$ chiral 
symmetry yields only two species. The shortcoming of this setup is the explicit breaking of part of the lattice discrete symmetry. The central-branch Wilson fermion [24, 32, 61-63] has

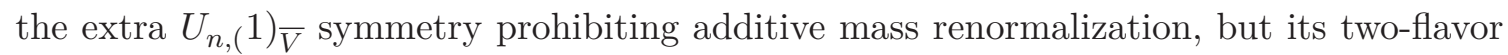
version loses part of hypercubic symmetry.

One of new avenues in the study of lattice fermions is to study fermions on non-torus lattices with different topologies. However, we are so far unfamiliar with the number of species on such lattices since the Nielsen-Ninomiya's no-go theorem can be only applied to the lattice fermions defined on the torus. ${ }^{1}$ Toward understanding the number of species of fermions on lattices with arbitrary topologies, we need to develop the way how to figure out the number of zero eigenvalues of lattice Dirac operators without using the momentum representation since the non-torus lattices do not have discrete translational symmetry.

In this paper, we discuss lattice fermions in terms of spectral graph theory (SGT) [6467] and obtain a new insight. The relation between supersymmetric lattice gauge theories (matrix models) and graph theories has been recently discussed [68, 69], and the relation among Dirac-Kaehler fermions, Euler characteristics of background spaces and quantum anomalies has been discussed in [70, 71]. We now focus on the spectral graph theory (SGT) and the discrete Fourier transformation (DFT), which tell us the relation among a graph, the associated matrix and its eigenvalues. We find that a Dirac operator of fermions defined on a certain lattice is identified as a matrix of the spectral graph corresponding to the lattice fermion. SGT and DFT clarifies the number of zero eigenvalues of lattice Dirac operators, leading to understanding of the number of doublers on lattices with arbitrary topologies. Our arguments are summarized as follows; first of all, a lattice fermion on a certain lattice corresponds to one spectral graph. Secondly, the Dirac operator of the lattice fermion can be treated as a matrix corresponding to the graph. Thus, once we have a certain lattice and a Dirac operator defined on the lattice, we can derive the corresponding matrix and find the number of zero eigenvalues by use of the discrete Fourier transformation (DFT). Finally, by taking an infinite-volume and continuum limits, we can study the number of doublers. We apply this procedure to the known fermion formulations including Naive fermion, Wilson fermion and Domain-wall fermion defined on the regular lattices, although the advantage of our technique is its use for non-torus lattices. We then apply it to the lattice fermion on the discretized four-dimensional hyperball and study the number of doublers on the bulk. We also discuss its application to hypersphere and discuss the possibility of a new theorem regarding the number of doublers on the lattice with arbitrary topologies.

The main purpose of this work is to introduce the importance of spectral graph theory to the lattice field theory community. Although most of the results obtained in this paper are reproductions of the known results, the method we use here is new and it could be applied to the nontrivial cases in future.

This paper is constructed as follows: in section 2 we introduce the spectral graph theory. In section 3 we discuss the naive fermion in terms of SGT and reproduce the known results. In section 4 and section 5 we discuss Wilson and domain-wall fermions

\footnotetext{
${ }^{1}$ Some may state that the Nielsen-Ninomiya's no-go theorem can be applied to fermions on non-torus lattices. However, this no-go theorem cannot explain the empirical fact that the number of fermion species is two on the discretized two-sphere.
} 

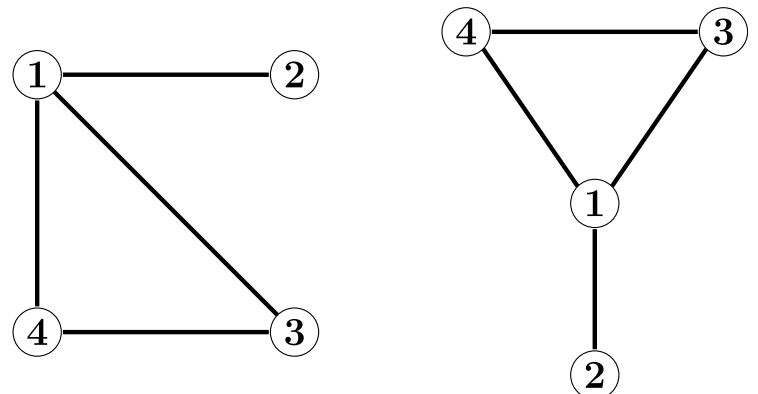

Figure 1. These examples are graphs having a pair $G=(V, E)$ with $V=\{1,2,3,4\}$ and $E=$ $\{\{1,2\},\{1,3\},\{1,4\},\{3,4\}\}$.

in terms of SGT and reproduce the known results. In section 6, as the first non-trivial application, we apply SGT analysis to the lattice fermions on four-dimensional hyperball. Section 7 is devoted to the summary and discussion.

\section{Spectral graph theory}

In the section, we introduce basic concepts of Spectral Graph Theory (SGT) for the purpose of discussing lattice fermions from a new viewpoint. We will also present some examples to show the importance of the concept. Let us start with the definition of a graph [64-67].

Definition 1 (graph) A graph $G$ is a pair $G=(V, E)$, where $V$ is a set of vertices of the graph and $E$ is a set of edges of the graph.

As examples, we exhibit two graphs in figure 1 with $V=\{1,2,3,4\}$ and $E=$ $\{\{1,2\},\{1,3\},\{1,4\},\{3,4\}\}$. Here, $\{i, j\}$ stands for an edge from $i$ to $j$. Note that two graphs in figure 1 have the common pair $G=(V, E)$ and they are the identical graphs although their appearances are different. They have no directed edges, which will be discussed soon, thus any two vertex in any element of $E$ in the examples can be commutated, so we find $\{i, j\}=\{j, i\}$.

Definition 2 (directed graph or digraph) A directed graph (or digraph) is a pair $(V, E)$ of sets of vertices and edges together with two maps init $: E \rightarrow V$ and ter $: E \rightarrow V$. The two maps are assigned to every edge $e_{i j}$ with an initial vertex $\operatorname{init}\left(e_{i j}\right)=v_{i} \in V$ and a terminal vertex $\operatorname{ter}\left(e_{i j}\right)=v_{j} \in V$. The edge $e_{i j}$ is said to be directed from $\operatorname{init}\left(e_{i j}\right)$ to $\operatorname{ter}\left(e_{i j}\right)$. If $\operatorname{init}\left(e_{i j}\right)=\operatorname{ter}\left(e_{i j}\right)$, the edge $e_{i j}$ is called a loop.

As examples, two graphs in figure 2 are digraphs with $V=\{1,2,3,4\}$ and $E=$ $\{\{1,2\},\{1,3\},\{1,4\},\{3,4\}\}$. Then initial vertex and terminal vertex are assigned as $\operatorname{init}(\{i, j\})=i, \operatorname{ter}(\{i, j\})=j$.

As another example we show figure 3, which is a digraph with loop. Note that vertices in any loop can be commutated since they are the identical vertex. In general, two vertices in a directed edge can not be commutated $\{i, j\} \neq\{j, i\}$. 

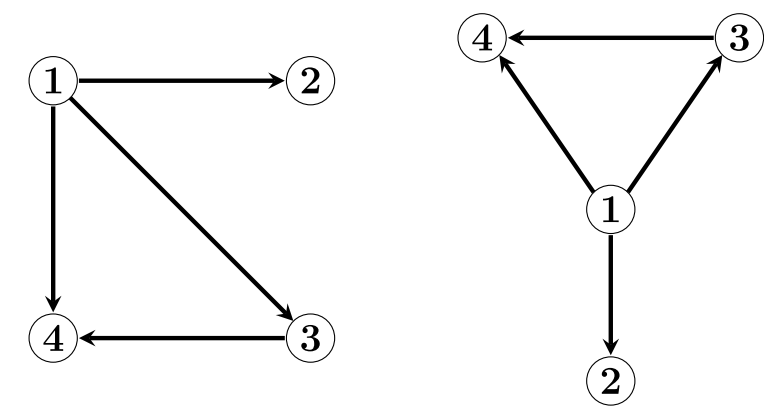

Figure 2. These examples are graphs having a pair $(V, E)$ with $V=\{1,2,3,4\}$ and $E=$ $\{\{1,2\},\{1,3\},\{1,4\},\{3,4\}\}$. The initial vertices of edges are $\operatorname{init}(\{1,2\})=1$, init $(\{1,3\})=$ 1 , init $(\{1,4\})=1, \operatorname{init}(\{3,4\})=3$, while the terminal vertices are $\operatorname{init}(\{1,2\})=2, \operatorname{init}(\{1,3\})=$ $3, \operatorname{init}(\{1,4\})=4, \operatorname{init}(\{3,4\})=4$.

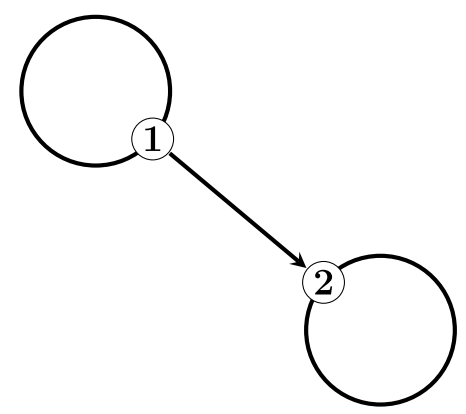

Figure 3. A digraph with two loops.

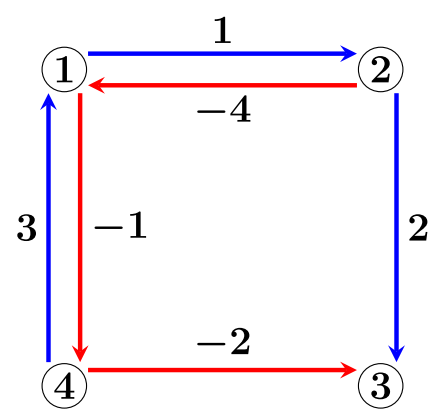

Figure 4. This digraph is a weighted graph. Blue edges in the graph are those with positive weights, while red edges are those with negative weights.

Definition 3 (weighted graph) The weighted graph has a value (the weight) for each edge in a graph or a digraph.

As an example, we show a digraph in figure 4. It is a weighted graph, each of whose edge has a weight.

Definition 4 (adjacency matrix) The adjacency matrix $A$ of a graph is the $|V| \times|V|$ 
matrix given by

$$
A_{i j}=\left\{\begin{array}{ll}
w_{i j} & \text { if there is a edge from } i \text { to } j \\
0 & \text { otherwise }
\end{array},\right.
$$

where $w_{i j}$ is the weight of an edge from $i$ to $j$.

As an example we exhibit an adjacency matrix $A$ of a graph in figure 4

$$
A=\left(\begin{array}{cccc}
0 & 1 & 0 & -1 \\
-4 & 0 & 2 & 0 \\
0 & 0 & 0 & 0 \\
3 & 0 & -2 & 0
\end{array}\right)
$$

This matrix is asymmetric. In general, the adjacency matrix of a directed graph is asymmetric since the existence of an edge from $i$ to $j$ does not necessarily imply that there is also an edge from $j$ to $i$.

The lattice fermion has common properties with the spectral graph we have introduced here. In the next sections, we will show that the lattice fermion can be identified as the spectral graph.

\section{Naive fermion}

In this section we discuss the Dirac operator matrix of Naive fermion in terms of SGT. Through this example, we will find a Dirac operator of lattice fermions is identified as a matrix of the spectral graph corresponding to the lattice fermion. We also show how to find the number of zero modes (the number of fermion species) by use of discrete Fourier transformation (DFT). This technique can be applied to any kind of matrices arising from the non-regular lattices. By use of DFT, we will correctly derive the sixteen fermion species in the naive fermion.

\subsection{Dirac matrix of naive fermion}

The lattice naive fermion action in four dimensions is

$$
S=\sum_{n} \sum_{\mu=1}^{4} \bar{\psi}_{n} \gamma_{\mu} D_{\mu} \psi_{n}
$$

where $D_{\mu} \equiv\left(T_{+\mu}-T_{-\mu}\right) / 2$ with $T_{ \pm \mu} \psi_{n}=U_{n, \pm \hat{\mu}} \psi_{n \pm \hat{\mu}}$ and $\hat{\mu}$ is a unit vector. In a free theory, we just set $U_{n, \pm \hat{\mu}}=\mathbf{1}$. The sum $\sum_{n}$ is the summation over lattice site $n=\left(n_{1}, n_{2}, n_{3}, n_{4}\right)$ and those intervals are $1 \leq n_{\mu} \leq N$. Note that the spacetime where the fermion is defined is a four-dimensional torus because we usually impose periodic boundary conditions in each direction. To derive the corresponding matrix of the lattice Dirac operator in a free theory, we introduce a vector of fermion fields. Namely, a vector $\psi$ is defined $\psi=\sum_{n} \psi_{n} \boldsymbol{e}_{n}$ 
where $\boldsymbol{e}_{n}$ are standard basis which satisfy orthonormal $\boldsymbol{e}_{n^{\prime}} \boldsymbol{e}_{n}=\delta_{n^{\prime} n} \equiv \prod_{i=1}^{4} \delta_{n_{i}^{\prime} n_{i}}$. $\delta_{k l}$ is the Kronecker delta. Here we specify that the order of components $\psi_{n}$ in the vector is $(1,1,1,1) \rightarrow \cdots \rightarrow(N, 1,1,1) \rightarrow(1,2,1,1) \rightarrow \cdots \rightarrow(N, N, 1,1) \rightarrow(1,1,2,1) \rightarrow \cdots \rightarrow$ $(N, N, N, N)$ in descending order. Namely,

$$
\psi=\left(\begin{array}{c}
\psi_{(1,1,1,1)} \\
\vdots \\
\psi_{(N, 1,1,1)} \\
\psi_{(1,2,1,1,1)} \\
\vdots \\
\psi_{(N, N, 1,1)} \\
\psi_{(1,1,2,1)} \\
\vdots \\
\psi_{(N, N, N, N)}
\end{array}\right)
$$

in term of the vector. Thus, the action of naive fermion can be rewritten as $S=\bar{\psi} \mathcal{D} \psi=$ $\sum_{m} \sum_{n} \bar{\psi}_{m} \mathcal{D}_{m n} \psi_{n}$ where $\mathcal{D}$ is Dirac matrix having $\mathcal{D}_{m n}$ as $(m, n)$ component. For later use, we now introduce the tensor-product representation: by the tensor product (or Kronecker product), this matrix can be represented as

$$
\begin{aligned}
\mathcal{D}= & \mathbf{1}_{N} \otimes \mathbf{1}_{N} \otimes \mathbf{1}_{N} \otimes P_{N} \otimes \gamma_{1} \\
& +\mathbf{1}_{N} \otimes \mathbf{1}_{N} \otimes P_{N} \otimes \mathbf{1}_{N} \otimes \gamma_{2} \\
& +\mathbf{1}_{N} \otimes P_{N} \otimes \mathbf{1}_{N} \otimes \mathbf{1}_{N} \otimes \gamma_{3} \\
& +P_{N} \otimes \mathbf{1}_{N} \otimes \mathbf{1}_{N} \otimes \mathbf{1}_{N} \otimes \gamma_{4}
\end{aligned}
$$

where $\mathbf{1}_{N}$ is an identity matrix of order $N$ and $P_{N}$ is a square matrix of order $N[72-$ 74]. The components of the matrix $P_{N}$ is defined as $\left(P_{N}\right)_{a b} \equiv\left(E_{a b}-E_{a b}^{-1}\right) / 2$ where $E_{a b} \equiv \sum_{i=1}^{N-1} \delta_{a i} \delta_{i+1 b}+\delta_{a N} \delta_{1 b}$ and $E_{a b}^{-1} \equiv \delta_{a 1} \delta_{N b}+\sum_{i=2}^{N} \delta_{a i} \delta_{i-1 b}$ for $a, b=1,2, \cdots, N . P_{N}$ is explicitly written as

$$
P_{N}=\frac{1}{2}\left(\begin{array}{ccccccc}
0 & 1 & 0 & & 0 & 0 & -1 \\
-1 & 0 & 1 & \cdots & 0 & 0 & 0 \\
0 & -1 & 0 & & 0 & 0 & 0 \\
& \vdots & & \ddots & & \vdots & \\
0 & 0 & 0 & & 0 & 1 & 0 \\
0 & 0 & 0 & \cdots & -1 & 0 & 1 \\
1 & 0 & 0 & & 0 & -1 & 0
\end{array}\right)
$$




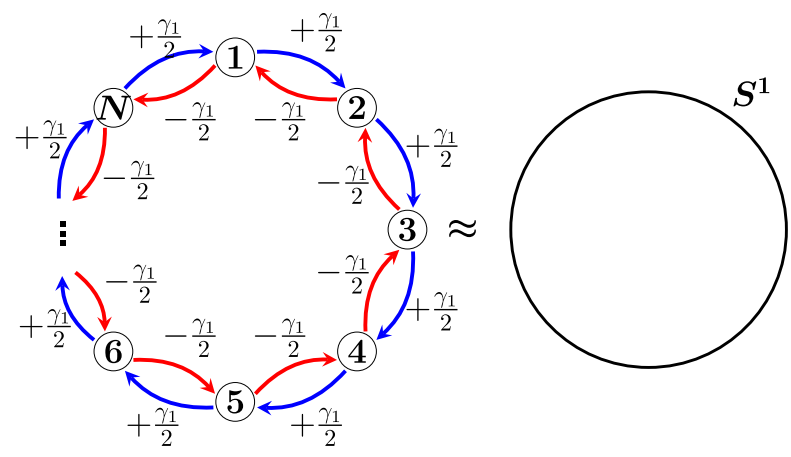

Figure 5. This graph corresponds to the Dirac matrix of one dimensional naive fermion with periodic boundary condition. This graph schematically shows a circle $S^{1}$.

and $E$ and $E^{\dagger}$ are similarly represented as

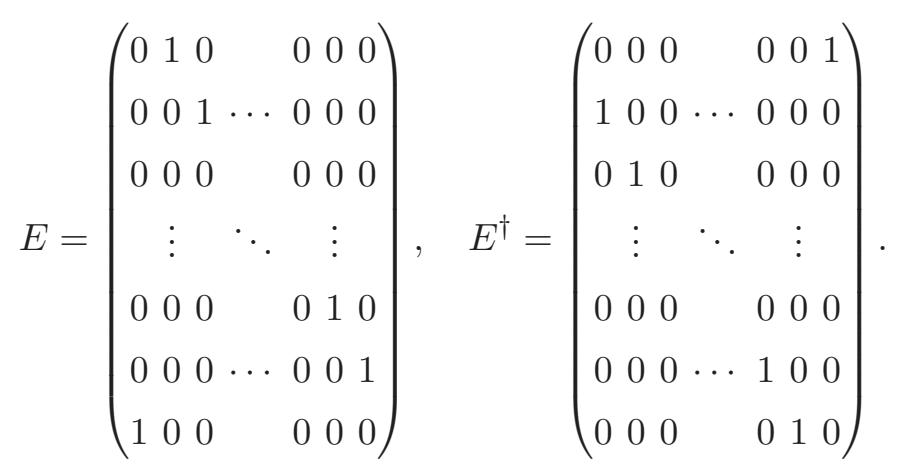

Note that the matrix $P_{N}$ is the matrix representing the periodic boundary condition of one direction. This matrix is the circulant matrix.

\subsection{Graphs corresponding to naive fermion}

In this subsection, based on the matrix representations we have obtained in the previous subsection, we see how the spectral graphs correspond to the naive lattice fermions on low- and high-dimensional lattices. Although in the cases of non-torus lattice fermions we obtain the Dirac matrix from the spectral graph, we now derive "the graph from the matrix" conversely.

Firstly, we consider the one dimensional lattice. In this case, the Dirac matrix of the free naive fermion is

$$
\mathcal{D}^{1 \mathrm{~d}}=P_{N} \otimes \gamma_{1}
$$

Now, the graph corresponding to this Dirac matrix is depicted in figure 5. This graph schematically represents a circle $S^{1}$, where we impose a periodic boundary condition. This is called a circulant graph in graph theory.

In the case of two-dimensional naive fermion, the Dirac matrix is

$$
D^{2 \mathrm{~d}}=\mathbf{1}_{N} \otimes P_{N} \otimes \gamma_{1}+P_{N} \otimes \mathbf{1}_{N} \otimes \gamma_{2},
$$

and the graph, who schematically represents two-dimensional torus $T^{2}$, is expressed as figure 6 , where each direction is independent, leading to $S^{1} \times S^{1} \sim T^{2}$. There are both an 


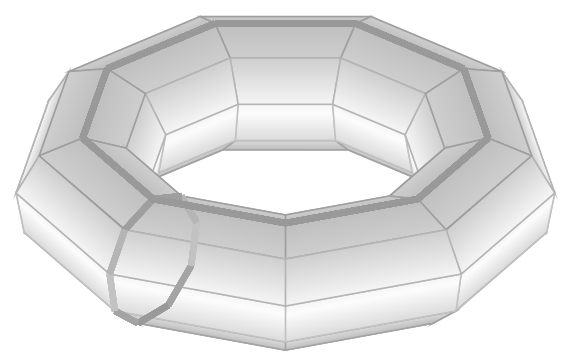

Figure 6. This graph corresponds to the Dirac matrix of two-dimensional naive fermion with periodic boundary condition. This graph schematically represents the two-dimensional torus $T^{2}$. The weight for each edge from $n$ to $n+\hat{\mu}$ is $+\gamma_{\mu} / 2$ and the one for each edge from $n$ to $n-\hat{\mu}$ is $-\gamma_{\mu} / 2$.

edge with the positive weight and an edge with the negative weight between each vertex. It is notable that the weight for edges from $n$ to $n+\hat{\mu}$ is $+\gamma_{\mu}$ and the one for edges from $n$ to $n-\hat{\mu}$ is $-\gamma_{\mu}$.

In three and four dimensions, the procedures are parallel. The corresponding graphs schematically represent $S^{1} \times S^{1} \times S^{1} \sim T^{3}$ and $S^{1} \times S^{1} \times S^{1} \times S^{1} \sim T^{4}$, respecctively. For the cases of non-torus lattice fermions, we conversely obtain the Dirac matrix from the spectral graph.

\subsection{Diagonalization of Dirac matrix $\mathcal{D}$}

In this subsection, we discuss how to diagonalize Dirac matrix $\mathcal{D}$ and find the number of zero eigenvalues for a free four-dimensional naive fermion. Firstly, we will diagonalize $E$ in order to diagonalize the whole Dirac matrix. We introduce the discrete Fourier transform (DFT) matrix $X$, which is consisted of $X_{j k} \equiv \xi^{(k-1)(j-1)} / \sqrt{N}$ with $\xi=e^{-\frac{2 \pi i}{N}}$ for $j, k=1,2, \cdots, N$. It is clear that DFT matrix is unitary. Then, $E$ and $E^{\dagger}$ satisfy $E X=X \Lambda$ and $E^{\dagger} X=\Lambda^{\dagger} X$, where $\Lambda$ is a diagonal matrix as $\Lambda=\operatorname{Diag}\left[1, \xi, \xi^{2}, \cdots, \xi^{N-1}\right]$. Since $P_{N}=E-E^{\dagger}$, the diagonalized $P_{N}$ is given as

$$
P_{N} X=i \operatorname{Diag}\left[0, \sin \frac{2 \pi}{N}, \sin \frac{4 \pi}{N}, \cdots, \sin \frac{2(N-1) \pi}{N}\right] X \equiv \Lambda_{P_{N}} X .
$$

There exist two zero eigenvalues in $\Lambda_{P_{N}}$ at most when $N$ is an even number. Now, the Dirac matrix $\mathcal{D}$ can be diagonalized as

$$
\begin{aligned}
\mathcal{U}^{\dagger} \mathcal{D} \mathcal{U}= & \mathbf{1}_{N} \otimes \mathbf{1}_{N} \otimes \mathbf{1}_{N} \otimes \Lambda_{P_{N}} \otimes \gamma_{1} \\
& +\mathbf{1}_{N} \otimes \mathbf{1}_{N} \otimes \Lambda_{P_{N}} \otimes \mathbf{1}_{N} \otimes \gamma_{2} \\
& +\mathbf{1}_{N} \otimes \Lambda_{P_{N}} \otimes \mathbf{1}_{N} \otimes \mathbf{1}_{N} \otimes \gamma_{3} \\
& +\Lambda_{P_{N}} \otimes \mathbf{1}_{N} \otimes \mathbf{1}_{N} \otimes \mathbf{1}_{N} \otimes \gamma_{4}
\end{aligned}
$$

where $\mathcal{U}$ is a unitary matrix defined as $\mathcal{U}=\bigotimes_{\mu=1}^{4} X \otimes \mathbf{1}_{4}$. It is easy to see the number zero eigenvalues of the Dirac matrix by introducing eigenvectors of $E$. We denote eigenvectors of $E$ as $|k\rangle$ for $k=1,2, \cdots, N$. They satisfy $E|k\rangle=\xi^{k-1}|k\rangle$ and $\left\langle k^{\prime} \mid k\right\rangle=\delta_{k^{\prime} k}$. Then, the unitary matrix $\mathcal{U}$ is written as $\mathcal{U}=\sum_{k_{4}, k_{3}, k_{2}, k_{1}}\left|k_{4}, k_{3}, k_{2}, k_{1}\right\rangle\left\langle k_{4}, k_{3}, k_{2}, k_{1}\right| \otimes \mathbf{1}_{4}$, where 
eigenvectors $\left|k_{4}, k_{3}, k_{2}, k_{1}\right\rangle$ mean $\left|k_{4}\right\rangle \otimes\left|k_{3}\right\rangle \otimes\left|k_{2}\right\rangle \otimes\left|k_{1}\right\rangle$. Thus the diagonalization of the Dirac matrix is expressed as

$$
\mathcal{U}^{\dagger} \mathcal{D} \mathcal{U}=\sum_{k_{4}, k_{3}, k_{2}, k_{1}}\left[i \sum_{\mu=1}^{4} \sin \left(\frac{2 \pi\left(k_{\mu}-1\right)}{N}\right) \gamma_{\mu}\right]\left|k_{4}, k_{3}, k_{2}, k_{1}\right\rangle\left\langle k_{4}, k_{3}, k_{2}, k_{1}\right| .
$$

If $\mathcal{U}^{\dagger} \mathcal{D} \mathcal{U}$ has zero eigenvalues, eq. (3.10) must satisfy an equation below

$$
\sum_{\mu=1}^{4} \sin \left(\frac{2 \pi\left(k_{\mu}-1\right)}{N}\right) \gamma_{\mu}=\mathbf{0}
$$

However, since $\gamma$ matrices are linearly independent, the coefficient of each $\gamma$ matrices must be zero. Finally, the conditions for the diagonalized Dirac matrix to have zero eigenvalues are

$$
\sin \left(\frac{2 \pi\left(k_{\mu}-1\right)}{N}\right)=0
$$

The solutions of eq. (3.12) are $k_{\mu}=1, \frac{N}{2}+1$. In this case, we do not need to take a continuum and a thermodynamic limit as long as we take an even number as $N$. Therefore, the number of zero eigenvalues are $2^{4}$ since the number of zero eigenvalues is equal to the number of combination of the solutions in four dimensions. This result is consistent with the well-known number of doublers in four-dimensional naive fermion.

In the end of this section, we comment on the meaning of $k_{\mu}$. This $k_{\mu}$ can be interpreted as a "shifted momentum" in this case. However, it is not necessarily equivalent to the momentum since it can be defined also on the lattice in which the momentum cannot be defined. Thus, $k_{\mu}$ should be simply interpreted as a variable for the diagonalization in general.

\section{Wilson fermion}

\subsection{Dirac matrix of Wilson fermion}

In this section we discuss the Dirac operator matrix of Wilson fermion in terms of SGT. The lattice Wilson fermion action in four dimensions is expressed as

$$
S_{W}=\sum_{n} \sum_{\mu} \bar{\psi}_{n} \gamma_{\mu} D_{\mu} \psi_{n}+m \sum_{n} \bar{\psi}_{n} \psi_{n}+r \sum_{n} \sum_{\mu} \bar{\psi}_{n}\left(1-C_{\mu}\right) \psi_{n}
$$

with $C_{\mu} \equiv\left(T_{+\mu}+T_{-\mu}\right) / 2 . m$ is a mass parameter and $r$ is a Wilson-fermion parameter. The periodic boundary condition is imposed on each direction and those intervals are given as $1 \leq n_{\mu} \leq N$. By investigating the action in a manner similar to section 3 , it is written as $S_{W}=\bar{\psi} \mathcal{D}_{W} \psi \equiv \bar{\psi}\left(\mathcal{D}+\mathcal{M}_{W}\right) \psi$, where $\mathcal{D}_{W}$ is the Dirac matrix of Wilson fermion and $\mathcal{M}_{W}$ is mass matrix consisting of the Wilson term. The mass matrix $\mathcal{M}_{W}$ is represented by use of tensor products as

$$
\begin{aligned}
\mathcal{M}_{W}= & m \cdot \mathbf{1}_{N} \otimes \mathbf{1}_{N} \otimes \mathbf{1}_{N} \otimes \mathbf{1}_{N} \otimes \mathbf{1}_{4} \\
+r \cdot & \left\{\mathbf{1}_{N} \otimes \mathbf{1}_{N} \otimes \mathbf{1}_{N} \otimes M_{W} \otimes \mathbf{1}_{4}+\mathbf{1}_{N} \otimes \mathbf{1}_{N} \otimes M_{W} \otimes \mathbf{1}_{N} \otimes \mathbf{1}_{4}\right. \\
& \left.\quad+\mathbf{1}_{N} \otimes M_{W} \otimes \mathbf{1}_{N} \otimes \mathbf{1}_{N} \otimes \mathbf{1}_{4}+M_{W} \otimes \mathbf{1}_{N} \otimes \mathbf{1}_{N} \otimes \mathbf{1}_{N} \otimes \mathbf{1}_{4}\right\}
\end{aligned}
$$

where $M_{W}=\mathbf{1}_{N}-\left(E+E^{\dagger}\right) / 2$ and the product $\cdot$ stands for scalar product. 


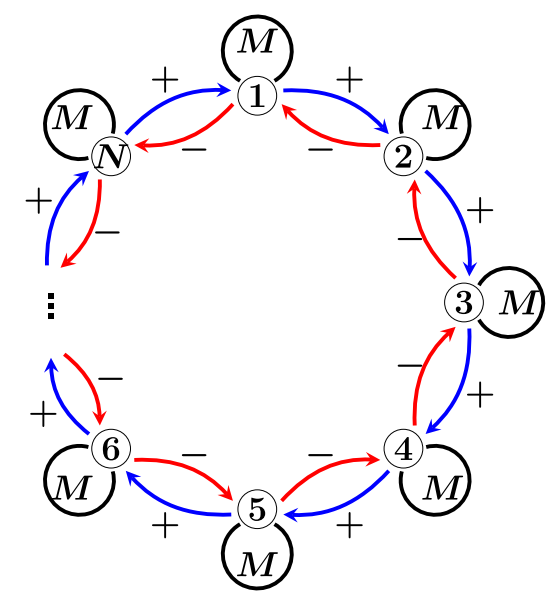

Figure 7. This digraph corresponds to the Dirac matrix of one-dimensional Wilson fermion. The weight of the loop is $M=m+r$. The weight \pm for each edge stand for $\pm\left(\gamma_{1} \mp r \mathbf{1}_{4}\right) / 2$.

\subsection{Graphs corresponding to Wilson fermion}

As with the case in section 3.2, we begin with the one-dimensional lattice. For onedimensional Wilson fermion, the Dirac matrix is given by

$$
D_{W}^{1 \mathrm{~d}}=P_{N} \otimes \gamma_{1}+m \mathbf{1}_{N} \otimes \mathbf{1}_{4}+r M_{W} \otimes \mathbf{1}_{4},
$$

and the graph corresponding to this matrix is depicted in figure 7. This graph again represents the circle $S^{1}$. However, the difference from the case of naive fermions in figure 6 is that figure 7 has a loop with the weight $M=m+r$ for each vertex.

In two dimensions, the Dirac matrix is

$$
\begin{aligned}
D_{W}^{2 \mathrm{~d}}= & \mathbf{1}_{N} \otimes P_{N} \otimes \gamma_{1}+P_{N} \otimes \mathbf{1}_{N} \otimes \gamma_{2} \\
& +m\left(\mathbf{1}_{N} \otimes \mathbf{1}_{N} \otimes \mathbf{1}_{4}\right)+r\left(\mathbf{1}_{N} \otimes M_{W}+M_{W} \otimes \mathbf{1}_{N}\right) \otimes \mathbf{1}_{4}
\end{aligned}
$$

and the graph corresponding to this matrix is given by figure 8 . The shape of the graph is topologically a two-dimensional torus $T^{2}$ however there is a loop with the weight $M=$ $m+\sum_{\mu} r$ for each vertex. Furthermore, the weight for each edge from $n$ to $n+\hat{1}$ is $+\left(\gamma_{1}-r \mathbf{1}_{4}\right) / 2$ and the one for each edge from $n$ to $n-\hat{1}$ is $-\left(\gamma_{1}+r \mathbf{1}_{4}\right) / 2$. In other direction $\mu=2$, we just replace $\gamma_{1}$ by $\gamma_{2}$.

The shape of the graph corresponding to Wilson fermion in four dimensions is again topologically a four-dimensional torus $T^{4}$. Furthermore, there is a loop for each vertex, which has the weight $M=m+\sum_{\mu} r$. The weight for each edge from $n$ to $n+\hat{\mu}$ is $+\left(\gamma_{\mu}-r \mathbf{1}_{4}\right) / 2$ while the wight for each edge from $n$ to $n-\hat{\mu}$ is $-\left(\gamma_{\mu}+r \mathbf{1}_{4}\right) / 2$.

\subsection{Diagonalization of Dirac matrix $\mathcal{D}_{W}$}

Firstly, we will diagonalize the mass matrix $\mathcal{M}_{W}$ so as to diagonalize the whole Dirac matrix $\mathcal{D}_{W}$. As with the discussion of naive fermion, we use the DFT matrix $X$. By using the matrix $X$ we can diagonalize $M_{W}$ as

$$
M_{W} X=\operatorname{Diag}\left[0,1-\cos \frac{2 \pi}{N}, 1-\cos \frac{4 \pi}{N}, \cdots, 1-\cos \frac{2(N-1) \pi}{N}\right] X \equiv \Lambda_{M_{W}} X .
$$




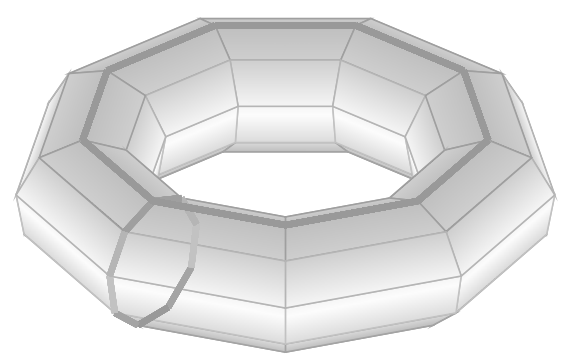

Figure 8. This digraph corresponds to the Dirac matrix in two-dimensional Wilson fermion. The shape of this graph is topologically equivalent to two-dimensional torus $T^{2}$. The weight for each edge from $n$ to $n+\hat{\mu}$ is $+\left(\gamma_{\mu}-r \mathbf{1}_{4}\right) / 2$ and the one for each edge from $n$ to $n-\hat{\mu}$ is $-\left(\gamma_{\mu}+r \mathbf{1}_{4}\right) / 2$. The weight for each loop is $M=m+\sum_{\mu} r$.

There is only a single zero eigenvalue in $\Lambda_{M_{W}}$ at most if $N$ is even number. As a result the diagonalization is expressed as

$$
\begin{aligned}
& \mathcal{U}^{\dagger} \mathcal{M}_{W} \mathcal{U}= m \cdot \mathbf{1}_{N} \otimes \mathbf{1}_{N} \otimes \mathbf{1}_{N} \otimes \mathbf{1}_{N} \otimes \mathbf{1}_{4} \\
&+r \cdot\left\{\mathbf{1}_{N} \otimes \mathbf{1}_{N} \otimes \mathbf{1}_{N} \otimes \Lambda_{M_{W}} \otimes \mathbf{1}_{4}+\mathbf{1}_{N} \otimes \mathbf{1}_{N} \otimes \Lambda_{M_{W}} \otimes \mathbf{1}_{N} \otimes \mathbf{1}_{4}\right. \\
&\left.\quad+\mathbf{1}_{N} \otimes \Lambda_{M_{W}} \otimes \mathbf{1}_{N} \otimes \mathbf{1}_{N} \otimes \mathbf{1}_{4}+\Lambda_{M_{W}} \otimes \mathbf{1}_{N} \otimes \mathbf{1}_{N} \otimes \mathbf{1}_{N} \otimes \mathbf{1}_{4}\right\}
\end{aligned}
$$

where $\mathcal{U}=\bigotimes_{\mu=1}^{4} X \otimes \mathbf{1}_{4}$. Since the Dirac matrix of naive fermion $\mathcal{D}$ can be diagonalized with the unitary matrix $U$, the Dirac matrix of Wilson fermion can be diagonalized as $\mathcal{U}^{\dagger} \mathcal{D}_{W} \mathcal{U}=\mathcal{U}^{\dagger} \mathcal{D} \mathcal{U}+\mathcal{U}^{\dagger} \mathcal{M}_{W} \mathcal{U}$. We introduce the eigenvectors $|k\rangle$ of $E$ to see the number of zero eigenvalues in the diagonalized Dirac matrix $\mathcal{U}^{\dagger} \mathcal{D}_{W} \mathcal{U}$. The diagonalized mass matrix $\mathcal{U}^{\dagger} \mathcal{M}_{W} \mathcal{U}$ is written as

$$
\begin{aligned}
\mathcal{U}^{\dagger} \mathcal{M}_{W} \mathcal{U}= & \sum_{k_{4}, k_{3}, k_{2}, k_{1}}\left[m \mathbf{1}_{4}+r \sum_{\mu=1}^{4}\left\{1-\cos \left(\frac{2 \pi\left(k_{\mu}-1\right)}{N}\right)\right\} \mathbf{1}_{4}\right] \\
& \times\left|k_{4}, k_{3}, k_{2}, k_{1}\right\rangle\left\langle k_{4}, k_{3}, k_{2}, k_{1}\right| .
\end{aligned}
$$

Thus the diagonalized Dirac matrix $\mathcal{U}^{\dagger} \mathcal{D}_{W} \mathcal{U}$ is

$$
\begin{aligned}
\mathcal{U}^{\dagger} \mathcal{D}_{W} \mathcal{U}= & \sum_{k_{4}, k_{3}, k_{2}, k_{1}}\left[i \sum_{\mu} \sin \left(\frac{2 \pi\left(k_{\mu}-1\right)}{N}\right) \gamma_{\mu}+r \sum_{\mu}\left\{\frac{m}{4 r}+1-\cos \left(\frac{2 \pi\left(k_{\mu}-1\right)}{N}\right)\right\} \mathbf{1}_{4}\right] \\
& \times\left|k_{4}, k_{3}, k_{2}, k_{1}\right\rangle\left\langle k_{4}, k_{3}, k_{2}, k_{1}\right| .
\end{aligned}
$$

If the diagonalized Dirac matrix $\mathcal{U}^{\dagger} \mathcal{D}_{W} \mathcal{U}$ has zero eigenvalues, eq. (4.8) must satisfy a equation

$$
i \sum_{\mu} \sin \left(\frac{2 \pi\left(k_{\mu}-1\right)}{N}\right) \gamma_{\mu}+r \sum_{\mu}\left\{\frac{m}{4 r}+1-\cos \left(\frac{2 \pi\left(k_{\mu}-1\right)}{N}\right)\right\} \mathbf{1}_{4}=\mathbf{0}
$$


Since $\gamma$ matrices and $\mathbf{1}_{4}$ are linearly independent, the conditions for the diagonalized Dirac matrix to have zero eigenvalues are given as

$$
\begin{aligned}
\sin \left(\frac{2 \pi\left(k_{\mu}-1\right)}{N}\right) & =0 \\
m+4 r-r \sum_{\mu} \cos \left(\frac{2 \pi\left(k_{\mu}-1\right)}{N}\right) & =0 .
\end{aligned}
$$

The solutions of eq. (4.10) are $k_{\mu}=1, \frac{N}{2}+1$. These solutions are classified by eq. (4.11) as

$$
M(k)= \begin{cases}m & \text { any } k_{\mu}=1 \text { in } k . \\ m+2 r & \text { one } k_{\mu}=1+N / 2 \text { otherwise } k_{\mu}=1 \text { in } k . \\ m+4 r & \text { two } k_{\mu}=1+N / 2 \text { otherwise } k_{\mu}=1 \text { in } k . \\ m+6 r & \text { three } k_{\mu}=1+N / 2 \text { otherwise } k_{\mu}=1 \text { in } k . \\ m+8 r & \text { any } k_{\mu}=1+N / 2 \text { in } k .\end{cases}
$$

where we define $M(k) \equiv m+4 r-r \sum_{\mu} \cos \left(\frac{2 \pi\left(k_{\mu}-1\right)}{N}\right)$ for $k=\left(k_{1}, k_{2}, k_{3}, k_{4}\right)$ in eq. (4.11). Hence, the number of zero eigenvalues of Dirac matrix $\mathcal{D}_{W}$ depends on mass parameter $m$ and Wilson-fermion parameter $r$. If we set $m=0$ and $r=1$, the number of zero eigenvalues is just one. In this case, we do not need to take a continuum and a thermodynamic limit as long as we take an even number as $N$. This result is consistent with the known result of Wilson fermion.

\section{Domain-wall fermion}

\subsection{Dirac matrix and mass matrix of domain-wall fermion}

In this section we discuss the Dirac operator matrix of domain-wall fermions in terms of SGT. The domain-wall fermion action in five dimensions ( $4+1$ dimensions) is

$$
\begin{aligned}
S_{D W}= & \sum_{n, s} \bar{\psi}_{n, s}\left[\sum_{\mu} \gamma_{\mu} D_{\mu} \psi_{n, s}+\gamma_{5} D_{s} \psi_{n, s}\right]-M_{0} \sum_{n, s} \bar{\psi}_{n, s} \psi_{n, s} \\
& +\sum_{n, s} \bar{\psi}_{n, s}\left[\sum_{\mu}\left(1-C_{\mu}\right) \psi_{n, s}+\left(1-C_{s}\right) \psi_{n, s}\right]
\end{aligned}
$$

where $D_{s} \psi_{n, s} \equiv\left(\psi_{n, s+1}-\psi_{n, s-1}\right) / 2, C_{s} \psi_{n, s} \equiv\left(\psi_{n, s+1}+\psi_{n, s-1}\right) / 2$ and $M_{0}$ is a mass parameter. The sum $\sum_{n, s}$ is the summation over five-dimensional lattice sites $n, s=$ $\left(n_{1}, n_{2}, n_{3}, n_{4} ; s\right)$. Note that there are two differences regarding boundary conditions and dynamical (or non-dynamical) variables between the fifth direction and other directions:

- On the fifth direction $s$, we impose the Dirichlet boundary condition, $\psi_{n, 0}=$ $\psi_{n, N_{s}+1}=0$, and its interval is $1 \leq s \leq N_{s}$. We impose a periodic boundary condition on other directions $n$ and those intervals are $1 \leq n_{\mu} \leq N$.

- The link variables in the fifth direction are not dynamical while those in other directions can be dynamical. 
Based on a similar discussion in section 3, the action of a free domain-wall fermion can be rewritten as $S_{D W}=\sum_{s, t} \bar{\psi}_{s}\left[\mathcal{D}_{D W}+\mathcal{M}_{D W}\right]_{s t} \psi_{t}$ for $s, t=1,2, \cdots, N_{s}$, where $\mathcal{D}_{D W}$ is the Dirac matrix of domain-wall fermion and $\mathcal{M}_{D W}$ is a mass matrix of domain-wall fermion from four-dimensional viewpoints. The Dirac matrix $\mathcal{D}_{D W}$ is

$$
\begin{aligned}
{\left[\mathcal{D}_{D W}\right]_{s t}=\delta_{s t} \cdot } & \left(\mathbf{1}_{N} \otimes \mathbf{1}_{N} \otimes \mathbf{1}_{N} \otimes P_{N} \otimes \gamma_{1}+\mathbf{1}_{N} \otimes \mathbf{1}_{N} \otimes P_{N} \otimes \mathbf{1}_{N} \otimes \gamma_{2}\right. \\
& \left.+\mathbf{1}_{N} \otimes P_{N} \otimes \mathbf{1}_{N} \otimes \mathbf{1}_{N} \otimes \gamma_{3}+P_{N} \otimes \mathbf{1}_{N} \otimes \mathbf{1}_{N} \otimes \mathbf{1}_{N} \otimes \gamma_{4}\right)
\end{aligned}
$$

and the mass matrix $\mathcal{M}_{D W}$ is

$$
\begin{aligned}
{\left[\mathcal{M}_{D W}\right]_{s t}=} & \frac{1}{2} \Delta_{s t}^{(-)} \cdot \mathbf{1}_{N} \otimes \mathbf{1}_{N} \otimes \mathbf{1}_{N} \otimes \mathbf{1}_{N} \otimes \gamma_{5} \\
& +\delta_{s t}\left(-M_{0} \cdot \mathbf{1}_{N} \otimes \mathbf{1}_{N} \otimes \mathbf{1}_{N} \otimes \mathbf{1}_{N} \otimes \mathbf{1}_{4}\right. \\
& \quad+\mathbf{1}_{N} \otimes \mathbf{1}_{N} \otimes \mathbf{1}_{N} \otimes M_{W} \otimes \mathbf{1}_{4}+\mathbf{1}_{N} \otimes \mathbf{1}_{N} \otimes M_{W} \otimes \mathbf{1}_{N} \otimes \mathbf{1}_{4} \\
& \left.\quad+\mathbf{1}_{N} \otimes M_{W} \otimes \mathbf{1}_{N} \otimes \mathbf{1}_{N} \otimes \mathbf{1}_{4}+M_{W} \otimes \mathbf{1}_{N} \otimes \mathbf{1}_{N} \otimes \mathbf{1}_{N} \otimes \mathbf{1}_{4}\right) \\
& +\frac{1}{2}\left(2 \delta_{s t}-\Delta_{s t}^{(+)}\right) \cdot \mathbf{1}_{N} \otimes \mathbf{1}_{N} \otimes \mathbf{1}_{N} \otimes \mathbf{1}_{N} \otimes \mathbf{1}_{4}
\end{aligned}
$$

where $\Delta_{s t}^{( \pm)}=\sum_{i=1}^{N_{s}-1} \delta_{s i} \delta_{i+1 t} \pm \sum_{l=2}^{N_{s}} \delta_{s l} \delta_{l-1 t}$. Note that we specify the order of components $\left(\psi_{n}\right)_{s}$ in the vector $\psi_{s}$ as $(1,1,1,1 ; s) \rightarrow \cdots \rightarrow(N, 1,1,1 ; s) \rightarrow(1,2,1,1 ; s) \rightarrow \cdots \rightarrow$ $(N, N, 1,1 ; s) \rightarrow(1,1,2,1 ; s) \rightarrow \cdots \rightarrow(N, N, N, N ; s)$ in a descending order. For simplicity, we introduce the chiral projection $\mathcal{P}_{R}$ and $\mathcal{P}_{L}$ for the mass matrix $\mathcal{M}_{D W}$. The matrix $\mathcal{M}_{D W}$ can then be rewritten as

$$
\begin{aligned}
{\left[\mathcal{M}_{D W}\right]_{s t}=} & \mathcal{M}_{s t}^{(L)} \otimes \mathcal{P}_{L}+\mathcal{M}_{s t}^{(R)} \otimes \mathcal{P}_{R} \\
\mathcal{M}_{s t}^{(L)}=\delta_{s t} & \left\{\left(1-M_{0}\right) \cdot \mathbf{1}_{N} \otimes \mathbf{1}_{N} \otimes \mathbf{1}_{N} \otimes \mathbf{1}_{N}\right. \\
& \quad+\mathbf{1}_{N} \otimes \mathbf{1}_{N} \otimes \mathbf{1}_{N} \otimes M_{W}+\mathbf{1}_{N} \otimes \mathbf{1}_{N} \otimes M_{W} \otimes \mathbf{1}_{N} \\
& \left.\quad+\mathbf{1}_{N} \otimes M_{W} \otimes \mathbf{1}_{N} \otimes \mathbf{1}_{N}+M_{W} \otimes \mathbf{1}_{N} \otimes \mathbf{1}_{N} \otimes \mathbf{1}_{N}\right\} \\
& -\sum_{i=1}^{N_{s}-1} \delta_{s i} \delta_{i+1 t} \cdot \mathbf{1}_{N} \otimes \mathbf{1}_{N} \otimes \mathbf{1}_{N} \otimes \mathbf{1}_{N}
\end{aligned}
$$

where $\mathcal{P}_{R} \equiv\left(\mathbf{1}_{4}+\gamma_{5}\right) / 2$ is a projection matrix into right-handed components and $\mathcal{P}_{L} \equiv$ $\left(\mathbf{1}_{4}-\gamma_{5}\right) / 2$ is into left-handed components.

\subsection{Graphs corresponding to domain-wall fermion}

We now discuss a graph corresponding to domain-wall fermion in two dimensions $(1+1$ dimensions). For two-dimensional domain-wall fermion, the Dirac matrix is given as

$$
\left[\mathcal{D}_{D W}^{1 \mathrm{~d}}\right]_{s t}=\delta_{s t} P_{N} \otimes \gamma_{1}
$$



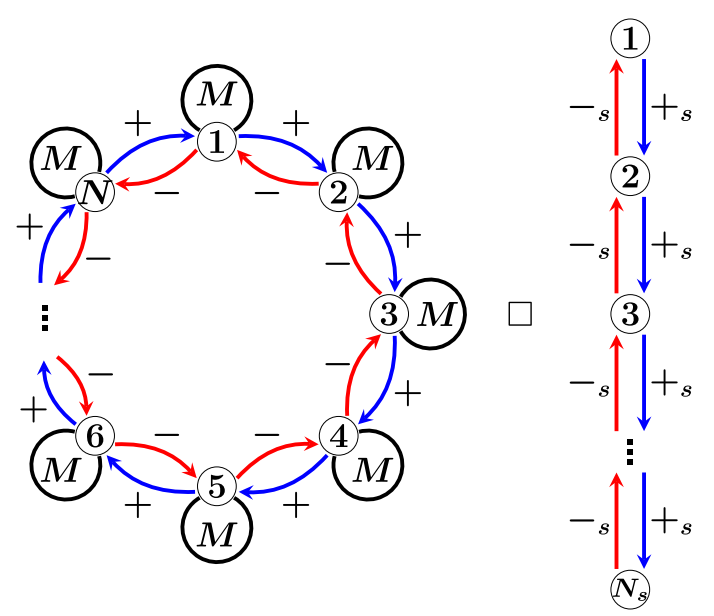

Figure 9. This digraph corresponds to the Dirac matrix and mass matrix in the two-dimensional domain-wall fermion. The left graph is equivalent to one-dimensional Wilson fermion. On the other hand, the right graph corresponds to the extra direction $s$. The weight ${ }_{s_{s}}$ means $+\left(\gamma_{3}-\mathbf{1}_{4}\right) / 2$ and $-_{s}$ means $-\left(\gamma_{3}+\mathbf{1}_{4}\right) / 2$. $\square$ stands for the Cartesian product.

and the mass matrix is

$$
\begin{aligned}
{\left[\mathcal{M}_{D W}^{1 \mathrm{~d}}\right]_{s t} } & =\frac{1}{2} \Delta_{s t}^{(-)} \cdot \mathbf{1}_{N} \otimes \gamma_{3}+\delta_{s t}\left(-M_{0} \mathbf{1}_{N} \otimes \mathbf{1}_{4}+M_{W} \otimes \mathbf{1}_{4}\right)+\frac{1}{2}\left(2 \delta_{s t}-\Delta_{s t}^{(+)}\right) \mathbf{1}_{N} \otimes \mathbf{1}_{4} \\
& =\mathcal{M}_{s t}^{1 \mathrm{~d}(L)} \otimes \mathcal{P}_{L}^{1 \mathrm{~d}}+\mathcal{M}_{s t}^{1 \mathrm{~d}(R)} \otimes \mathcal{P}_{R}^{1 \mathrm{~d}}
\end{aligned}
$$

where

$$
\begin{aligned}
\mathcal{M}_{s t}^{1 \mathrm{~d}(L)} & =\delta_{s t}\left\{\left(1-M_{0}\right) \mathbf{1}_{N}+M_{W}\right\}-\sum_{i=1}^{N_{s}-1} \delta_{s i} \delta_{i+1 t} \mathbf{1}_{N} \\
\mathcal{M}_{s t}^{1 \mathrm{~d}(R)} & =\delta_{s t}\left\{\left(1-M_{0}\right) \mathbf{1}_{N}+M_{W}\right\}-\sum_{l=2}^{N_{s}} \delta_{s l} \delta_{l-1 t} \mathbf{1}_{N} \\
\mathcal{P}_{L}^{1 \mathrm{~d}} & =\frac{1}{2}\left(\mathbf{1}_{4}-\gamma_{3}\right), \quad \mathcal{P}_{R}^{1 \mathrm{~d}}=\frac{1}{2}\left(\mathbf{1}_{4}+\gamma_{3}\right) .
\end{aligned}
$$

We adopt $\gamma_{3}$ as the extra dimensional gamma matrix. A graph corresponding to the Dirac matrices is figure 9. The schematicl shape of this graph is paetially similar to the graph in one-dimensional Wilson fermion. There is also a loop with the weight $M=2-M_{0}$ for each vertex. Each edge from $n$ to $n+\hat{1}$ has the weight $+\left(\gamma_{1}-\mathbf{1}_{4}\right) / 2$ and the one from $n$ to $n-\hat{1}$ has the weight $-\left(\gamma_{1}+\mathbf{1}_{4}\right) / 2$. However, there is another direction $s$ and each edge from $s$ to $s \pm 1$ has the weight $\pm\left(\gamma_{5} \mp \mathbf{1}_{4}\right) / 2$. The two graphs corresponding to the direction $\mu=1$ and in the extra direction $s$ is producted by Cartesian product $\square$. It is notable that a graph in the direction $\mu=1$ is equivalent to a graph in one-dimensional Wilson fermion.

For the five-dimensional domain-wall fermion, a graph also consists of the two graphs, a graph corresponding to the four dimensional Wilson fermion and a graph in the extra direction $s$, which are unified by Cartesian product. 


\begin{tabular}{|lcc|}
\hline the solutions of $k_{\mu}$ & the range of $M_{0}$ & the number of zero eigenvalues \\
\hline any $k_{\mu}=1$ & $0<M_{0}<2$ & 1 \\
one $k_{\mu}=1+N / 2$ otherwise $k_{\mu}=1$ & $2<M_{0}<4$ & 4 \\
two $k_{\mu}=1+N / 2$ otherwise $k_{\mu}=1$ & $4<M_{0}<6$ & 6 \\
three $k_{\mu}=1+N / 2$ otherwise $k_{\mu}=1$ & $6<M_{0}<8$ & 4 \\
any $k_{\mu}=1+N / 2$ & $8<M_{0}<10$ & 1 \\
\hline
\end{tabular}

Table 1. Classification of the number of zero eigenvalues in DW fermion.

\subsection{Diagonalization of Dirac matrix $\mathcal{D}_{D W}$ and mass matrix $\mathcal{M}_{D W}$}

Firstly, We diagonalize the Dirac matrix $\mathcal{D}_{D W}$ in a parallel way to section 3 . By using the unitary matrix $\mathcal{U}$, the diagonalized Dirac matrix is given as

$$
\left[\mathcal{U}^{\dagger} \mathcal{D}_{D W} \mathcal{U}\right]_{s t}=\delta_{s t} \sum_{k_{4}, k_{3}, k_{2}, k_{1}}\left[i \sum_{\mu=1}^{4} \sin \left(\frac{2 \pi\left(k_{\mu}-1\right)}{N}\right) \gamma_{\mu}\right]\left|k_{4}, k_{3}, k_{2}, k_{1}\right\rangle\left\langle k_{4}, k_{3}, k_{2}, k_{1}\right|
$$

for $k_{\mu}=1,2, \cdots, N$. As a result, the solutions of $k_{\mu}$ for the Dirac matrix $\mathcal{D}_{D W}$ to have zero eigenvalues are given by $k_{\mu}=1,1+N / 2$.

Secondly, we will diagonalize the mass matrix $\mathcal{M}_{D W}$. The diagonalized mass matrix is

$$
\begin{aligned}
{\left[\mathcal{U}^{\dagger} \mathcal{M}_{D W} \mathcal{U}\right]_{s t}=} & \mathcal{U}^{\dagger}\left(\mathcal{M}_{s t}^{(L)} \otimes \mathcal{P}_{L}\right) \mathcal{U}+\mathcal{U}^{\dagger}\left(\mathcal{M}_{s t}^{(R)} \otimes \mathcal{P}_{R}\right) \mathcal{U} \\
= & \sum_{k_{4}, k_{3}, k_{2}, k_{1}}\left[\left\{W(k) \delta_{s t}-\sum_{i=1}^{N_{s}-1} \delta_{s i} \delta_{i+1 t}\right\} \mathcal{P}_{L}+\left\{W(k) \delta_{s t}-\sum_{l=2}^{N_{s}} \delta_{s l} \delta_{l-1 t}\right\} \mathcal{P}_{R}\right] \\
& \times\left|k_{4}, k_{3}, k_{2}, k_{1}\right\rangle\left\langle k_{4}, k_{3}, k_{2}, k_{1}\right|
\end{aligned}
$$

where $W(k) \equiv 1-M_{0}+\sum_{\mu}\left\{1-\cos \left(\frac{2 \pi\left(k_{\mu}-1\right)}{N}\right)\right\}$. We now impose conditions, $|W(k)|<$ 1and $N_{s}=\infty$ so that mass matrix $\mathcal{M}_{D W}$ has zero eigenvalues. We can determine the range of mass parameter $M_{0}$ from $|W(k)|<1$. Using the solutions of $k_{\mu}, W(k)$ can be classified as

$$
W(k)= \begin{cases}1-M_{0} & \text { any } k_{\mu}=1 \text { in } k . \\ 3-M_{0} & \text { one } k_{\mu}=1+N / 2 \text { otherwise } k_{\mu}=1 \text { in } k . \\ 5-M_{0} & \text { two } k_{\mu}=1+N / 2 \text { otherwise } k_{\mu}=1 \text { in } k . \\ 7-M_{0} & \text { three } k_{\mu}=1+N / 2 \text { otherwise } k_{\mu}=1 \text { in } k . \\ 9-M_{0} & \text { any } k_{\mu}=1+N / 2 \text { in } k .\end{cases}
$$

Therefore, the relation between the solutions of $k_{\mu}$, the range of mass parameter $M_{0}$, and the number of zero eigenvalues are given in table. 1 . The result is consistent with the known results of the domain-wall fermion. It indicates that our method can be applied to the lattice fermion with Dirichlet boundary conditions. 


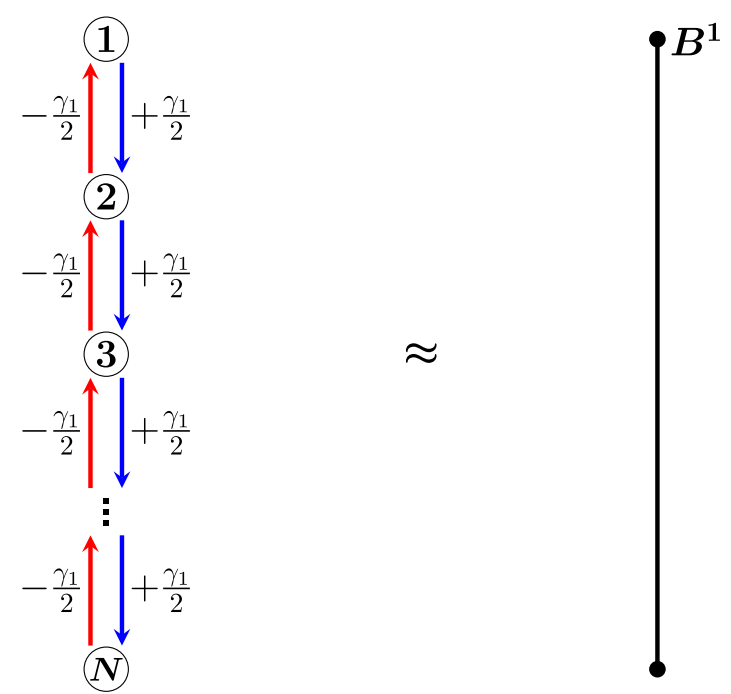

Figure 10. This digraph corresponds to the lattice fermion on the discretized $B^{1}$.

\section{Lattice fermions on four dimensional hyperball}

In section $3,4,5$, we show that there are the spectral digraphs corresponding to the Dirac matrices of the naive fermion, Wilson fermion, and domain-wall fermion. Then, we find out the number of fermion species based on the discrete Fourier transformation.

Our question is whether we can obtain the Dirac matrix when the spectral digraph corresponding to a lattice fermion is given. In this section, we will construct the Dirac matrix from a digraph corresponding to a certain lattice fermion and derive the number of fermion species by use of DFT. Through this procedure, we can construct the Dirac matrix for a lattice fermion defined on complicated lattices, where the momentum cannot be defined. Then, we can derive the number of the species for fermions on such lattices.

From the next subsection we consider the discretized four-dimensional hyperball $B^{4}$. This space is obtaining by imposing the Dirichlet boundary condition on each direction of four dimensions.

\subsection{Graphs corresponding to the Dirac matrix on hyperball}

We discuss the graph corresponding to the fermion defined on the four-dimensional hyperball. For simplicity, we first study a graph corresponding to lattice fermion on the discretized one-dimensional ball $B^{1}$ (or line segment). The digraph corresponding to the discretized $B^{1}$ is given as figure 10. Note that the hopping between two ends in position space is prohibited. The Dirac matrix corresponding to this graph is given by

$$
\mathcal{D}_{B^{1}}=Q_{N} \otimes \gamma_{1}
$$




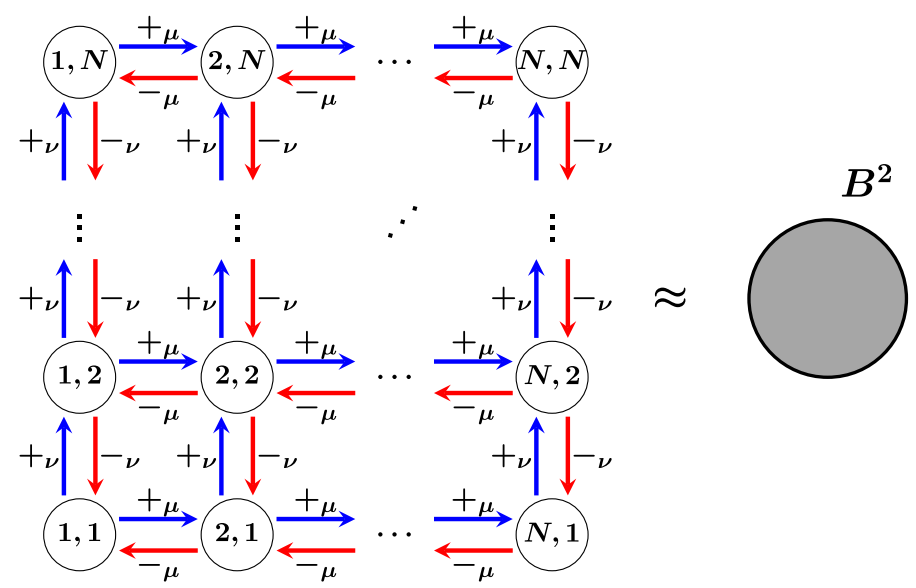

Figure 11. This digraph corresponds to fermions on the discretized $B^{2}$. As with the onedimensional case, the weight of blue edge is $+\gamma_{\mu} / 2$ and the one of the red edge is $-\gamma_{\mu} / 2$.

where $\left(Q_{N}\right)_{a b} \equiv\left(\sum_{i=1}^{N-1} \delta_{a i} \delta_{i+1 b}-\sum_{l=2}^{N} \delta_{a l} \delta_{l-1 b}\right) / 2$. This matrix $Q_{N}$ is written as

$$
Q_{N}=\frac{1}{2}\left(\begin{array}{ccccccc}
0 & 1 & 0 & & 0 & 0 & 0 \\
-1 & 0 & 1 & \cdots & 0 & 0 & 0 \\
0 & -1 & 0 & & 0 & 0 & 0 \\
& \vdots & & \ddots & & \vdots & \\
0 & 0 & 0 & & 0 & 1 & 0 \\
0 & 0 & 0 & \cdots & -1 & 0 & 1 \\
0 & 0 & 0 & & 0 & -1 & 0
\end{array}\right)
$$

The prohibition of hopping in the two ends is represented by the two elements $(1, N)$ and $(N, 1)$ which are zero in this case.

For the discretized two-dimensional ball $B^{2}$ (or disk), the digraph is given as figure 11 . The Dirac matrix in two dimensions is given by

$$
\mathcal{D}_{B^{2}}=\mathbf{1}_{N} \otimes Q_{N} \otimes \gamma_{1}+Q_{N} \otimes \mathbf{1}_{N} \otimes \gamma_{2}
$$

As seen from figures 10 and 11, the translational symmetry is partially broken since there is a boundary.

Through a parallel discussion, we find the shape of the digraph corresponding to fermions on the discretized three dimensional ball $B^{3}$ become a box, where the sites in this space is expressed by $\left(n_{1}, n_{2}, n_{3}\right)$ whith $1 \leq n_{i} \leq N$. The Dirac matrix on the discretized $B^{3}$ is given as

$$
\mathcal{D}_{B^{3}}=\mathbf{1}_{N} \otimes \mathbf{1}_{N} \otimes Q_{N} \otimes \gamma_{1}+\mathbf{1}_{N} \otimes Q_{N} \otimes \mathbf{1}_{N} \otimes \gamma_{2}+Q_{N} \otimes \mathbf{1}_{N} \otimes \mathbf{1}_{N} \otimes \gamma_{3} .
$$

In a similar manner we can construct the digraph and the corresponding Dirac matrix on the discretized four dimensional hyperball $B^{4}$. In the next subsection, we will investigate the lattice action on the discretized $B^{4}$. 


\subsection{Lattice action on four-dimensional hyperball}

The free naive fermion action on four-dimensional discretized hyperball $B^{4}$ is

$$
S_{B^{4}}=\sum_{n} \sum_{\mu} \bar{\psi}_{n} \gamma_{\mu} D_{\mu} \psi_{n}
$$

where the lattice sites are $n=\left(n_{1}, n_{2}, n_{3}, n_{4}\right)$ for $1 \leq n_{i} \leq N$ and Dirichlet boundary conditions are imposed on each direction. This action is also expressed as $S_{B^{4}}=\bar{\psi} \mathcal{D}_{B^{4}} \psi$. The Dirac matrix $\mathcal{D}_{B^{4}}$ is

$$
\begin{aligned}
\mathcal{D}_{B^{4}}= & \mathbf{1}_{N} \otimes \mathbf{1}_{N} \otimes \mathbf{1}_{N} \otimes Q_{N} \otimes \gamma_{1} \\
& +\mathbf{1}_{N} \otimes \mathbf{1}_{N} \otimes Q_{N} \otimes \mathbf{1}_{N} \otimes \gamma_{2} \\
& +\mathbf{1}_{N} \otimes Q_{N} \otimes \mathbf{1}_{N} \otimes \mathbf{1}_{N} \otimes \gamma_{3} \\
& +Q_{N} \otimes \mathbf{1}_{N} \otimes \mathbf{1}_{N} \otimes \mathbf{1}_{N} \otimes \gamma_{4} .
\end{aligned}
$$

\subsection{Number of species on four-dimensional hyperball}

Firstly, we will discuss diagonalization of the matrix $Q_{N}$ so as to derive the number of species on $B^{4}$. This matrix can be diagonalized by use of a unitary matrix $Y$ defined as $(Y)_{a b}=\alpha i^{b} \sin \left(\frac{a b \pi}{N+1}\right)$, where $\alpha$ is a normalization coefficient $[75,76]$. By using the unitary matrix, the diagonalized matrix is given as

$$
Q_{N} Y=i \operatorname{Diag}\left[\cos \left(\frac{\pi}{N+1}\right), \cos \left(\frac{2 \pi}{N+1}\right), \cdots, \cos \left(\frac{N \pi}{N+1}\right)\right] \equiv \Lambda_{Q_{N}} X .
$$

Note that there is a single zero eigenvalue in $\Lambda_{Q_{N}}$ at most when the number of sites in each direction $N$ is odd number. The Dirac matrix $\mathcal{D}_{B^{4}}$ is diagonalized as

$$
\begin{aligned}
\mathcal{V}^{\dagger} \mathcal{D}_{B^{4}} \mathcal{V}= & \mathbf{1}_{N} \otimes \mathbf{1}_{N} \otimes \mathbf{1}_{N} \otimes \Lambda_{Q_{N}} \otimes \gamma_{1} \\
& +\mathbf{1}_{N} \otimes \mathbf{1}_{N} \otimes \Lambda_{Q_{N}} \otimes \mathbf{1}_{N} \otimes \gamma_{2} \\
& +\mathbf{1}_{N} \otimes \Lambda_{Q_{N}} \otimes \mathbf{1}_{N} \otimes \mathbf{1}_{N} \otimes \gamma_{3} \\
& +\Lambda_{Q_{N}} \otimes \mathbf{1}_{N} \otimes \mathbf{1}_{N} \otimes \mathbf{1}_{N} \otimes \gamma_{4}
\end{aligned}
$$

where $\mathcal{V}$ is a unitary matrix defined as $\mathcal{V} \equiv \otimes_{\mu=1}^{4} Y \otimes \mathbf{1}_{4}$. To study eigenvalues in the Dirac matrix $\mathcal{D}_{B^{4}}$, we introduce eigenvector $|\lambda\rangle$ satisfying $Q_{N}|\lambda\rangle=i \cos \left(\frac{\lambda \pi}{N+1}\right)|\lambda\rangle$ for $\lambda=1,2, \cdots, N$. The diagonalized Dirac matrix is then written

$$
\mathcal{V}^{\dagger} \mathcal{D}_{B^{4}} \mathcal{V}=\sum_{\lambda_{1}, \lambda_{2}, \lambda_{3}, \lambda_{4}}\left[i \sum_{\mu=1}^{4} \cos \left(\frac{\lambda_{\mu} \pi}{N+1}\right) \gamma_{\mu}\right]\left|\lambda_{1}, \lambda_{2}, \lambda_{3}, \lambda_{4}\right\rangle\left\langle\lambda_{1}, \lambda_{2}, \lambda_{3}, \lambda_{4}\right|
$$

where $\left|\lambda_{1}, \lambda_{2}, \lambda_{3}, \lambda_{4}\right\rangle=\left|\lambda_{1}\right\rangle \otimes\left|\lambda_{2}\right\rangle \otimes\left|\lambda_{3}\right\rangle \otimes\left|\lambda_{4}\right\rangle$. For the diagonal matrix $\mathcal{V}^{\dagger} \mathcal{D}_{B^{4}} \mathcal{V}$ to have zero eigenvalues, the following equation must be satisfied,

$$
\sum_{\mu} \cos \left(\frac{\lambda_{\mu} \pi}{N+1}\right) \gamma_{\mu}=0 .
$$


Furthermore, since $\gamma$ matrices is linearly independent, this equation is rewritten as

$$
\cos \left(\frac{\lambda_{\mu} \pi}{N+1}\right)=0
$$

The solution of eq. (6.11) is $\lambda_{\mu}=\frac{N+1}{2}$. If the number of sites in the each direction is even, there is no zero eigenvalues in the Dirac matrix. As a result, there are no solutions $\lambda_{\mu}$ both satisfying eq. (6.11) and belonging to the set of integer, $\{1,2, \cdots, N\}$. On the other hand, when $N$ is the odd number, there is a single zero eigenvalue. Therefore, there is one physical pole on the bulk of four-dimensional hyperball when the number of sites in each direction is the odd number. If we take a thermodynamical limit for even $N$, one of the non-zero eigenvalue approaches to zero. This result is true in any dimensional lattice since $\gamma$ matrices in any dimension is linearly independent. Thus, lattice fermions on the finite-volume lattice of $d$-dimensional hyperball $B^{d}$ have one physical pole on the bulk.

As is well-known, the lattice fermion defined on a lattice with boundaries can have edge modes on the boundaries. Therefore, the edge mode works to cancel the gauge anomaly at the boundary when we introduce gauge fields or link variables. The existence of a single Dirac mode on the bulk in the present lattice fermion with boundaries is reasonable as with the case of the domain-wall fermion.

The results we have obtained in this section are again not so novel. However, we have shown that we can easily find the number of zero eigenvalues or the number of species by obtaining the spectral digraph corresponding to the lattice fermions and taking the procedure of discrete Fourier transformation.

\section{Summary and discussion}

In this note, we have introduced a new insight into lattice fermions based on spectral graph theory (SGT). By use of SGT and discrete Fourier transformation (DFT), we can figure out the number of zero eigenvalues of lattice Dirac operators without considering the momentum space. Then, we can understand the number of doublers on non-torus lattices with arbitrary topologies. The most important fact in this analysis is that a lattice fermion on a certain lattice corresponds to one spectral graph. Then, Dirac operators of lattice fermions can be treated as matrices corresponding to graphs. Once we have a certain lattice and a Dirac operator defined on the lattice, we can derive the corresponding matrix and find the number of zero eigenvalues by use of the discrete Fourier transformation (DFT), leading to understanding of the number of doublers. We have applied this procedure to the known fermion formulations including Naive fermion, Wilson fermion and Domainwall fermion, and discuss the number of doublers in terms of SGT. As the first non-trivial application, we have studied lattice fermions on the discretized hyperball and discussed the number of fermion species on the bulk.

We now discuss further application of SGT to lattice fermions;

1. Application to discretized sphere: it is possible to apply SGT to lattice fermions on discretized spheres via the method in section 6 . The $d$ dimensional sphere can be realized by eliminating the bulk of $d+1$ dimensional hyperball $B^{d+1}$. By using SGT, 
the Dirac matrix in this situation is simply obtained since what we have to do is to eliminate the matrix elements corresponding to the bulk. We will discuss this case in the future work soon.

2. A new theorem on the lattice with arbitrary Euler characteristics: the method based on SGT can be applied to the lattices with arbitrary Euler characteristics since we simply construct the graphs corresponding discretized manifolds with arbitrary topology. As a result, we may be able to conjecture a new theorem which tells us the number of species on more generic lattices by studying lattice fermions based on SGT. One of keys for such a theorem is the topology of the graph itself. We will also discuss this point in the future work soon.

3. Novel lattice fermions: we can propose novel lattice fermions by translating a matrix with desirable properties (minimal zero modes, hermiticity or chirality) to a spectral graph, which corresponds to the lattice fermion. A fermion obtained by this procedure may correspond to a lattice fermion defined on non-torus lattice.

\section{Acknowledgments}

T. M. is grateful to K. Ohta, S. Matsuura and S. Kamata for the fruitful discussion on the relation between graph theory and lattice field theory. This work of T. M. is supported by the Japan Society for the Promotion of Science (JSPS) Grant-in-Aid for Scientific Research (KAKENHI) Grant Numbers 19K03817 and 18H01217.

Open Access. This article is distributed under the terms of the Creative Commons Attribution License (CC-BY 4.0), which permits any use, distribution and reproduction in any medium, provided the original author(s) and source are credited.

\section{References}

[1] K.G. Wilson, Confinement of quarks, Phys. Rev. D 10 (1974) 2445 [InSPIRE].

[2] M. Creutz, Monte Carlo study of quantized SU(2) gauge theory, Phys. Rev. D 21 (1980) 2308 [INSPIRE].

[3] L.H. Karsten and J. Smit, Lattice fermions: species doubling, chiral invariance, and the triangle anomaly, Nucl. Phys. B 183 (1981) 103 [INSPIRE].

[4] H.B. Nielsen and M. Ninomiya, Absence of neutrinos on a lattice. 1. Proof by homotopy theory, Nucl. Phys. B 185 (1981) 20 [Erratum ibid. 195 (1982) 541] [INSPIRE].

[5] H.B. Nielsen and M. Ninomiya, Absence of neutrinos on a lattice. 2. Intuitive topological proof, Nucl. Phys. B 193 (1981) 173 [INSPIRE].

[6] H.B. Nielsen and M. Ninomiya, No go theorem for regularizing chiral fermions, Phys. Lett. B 105 (1981) 219 [INSPIRE].

[7] K.G. Wilson, Quarks and strings on a lattice, in New phenomena in subnuclear physics. Proceedings, International School of Subnuclear Physics, Erice, Sicily, Italy 11 July-1 August 1975. Part A, Springer, Boston, MA, U.S.A. (1977), pg. 69. 
[8] D.B. Kaplan, A method for simulating chiral fermions on the lattice, Phys. Lett. B 288 (1992) 342 [hep-lat/9206013] [INSPIRE].

[9] Y. Shamir, Chiral fermions from lattice boundaries, Nucl. Phys. B 406 (1993) 90 [hep-lat/9303005] [INSPIRE].

[10] V. Furman and Y. Shamir, Axial symmetries in lattice QCD with Kaplan fermions, Nucl. Phys. B 439 (1995) 54 [hep-lat/9405004] [INSPIRE].

[11] H. Neuberger, More about exactly massless quarks on the lattice, Phys. Lett. B 427 (1998) 353 [hep-lat/9801031] [INSPIRE].

[12] P.H. Ginsparg and K.G. Wilson, A remnant of chiral symmetry on the lattice, Phys. Rev. D 25 (1982) 2649 [inSPIRE].

[13] J.B. Kogut and L. Susskind, Hamiltonian formulation of Wilson's lattice gauge theories, Phys. Rev. D 11 (1975) 395 [InSPIRE].

[14] L. Susskind, Lattice fermions, Phys. Rev. D 16 (1977) 3031 [INSPIRE].

[15] N. Kawamoto and J. Smit, Effective Lagrangian and dynamical symmetry breaking in strongly coupled lattice QCD, Nucl. Phys. B 192 (1981) 100 [INSPIRE].

[16] H.S. Sharatchandra, H.J. Thun and P. Weisz, Susskind fermions on a Euclidean lattice, Nucl. Phys. B 192 (1981) 205 [inSPIRE].

[17] M.F.L. Golterman and J. Smit, Selfenergy and flavor interpretation of staggered fermions, Nucl. Phys. B 245 (1984) 61 [INSPIRE].

[18] M.F.L. Golterman, Staggered mesons, Nucl. Phys. B 273 (1986) 663 [InSPIRE].

[19] G.W. Kilcup and S.R. Sharpe, A tool kit for staggered fermions, Nucl. Phys. B 283 (1987) 493 [INSPIRE].

[20] W. Bietenholz and I. Hip, The scaling of exact and approximate Ginsparg-Wilson fermions, Nucl. Phys. B $\mathbf{5 7 0}$ (2000) 423 [hep-lat/9902019] [INSPIRE].

[21] M. Creutz, T. Kimura and T. Misumi, Index theorem and overlap formalism with naive and minimally doubled fermions, JHEP 12 (2010) 041 [arXiv: 1011.0761] [INSPIRE].

[22] S. Dürr and G. Koutsou, Brillouin improvement for Wilson fermions, Phys. Rev. D 83 (2011) 114512 [arXiv:1012.3615] [INSPIRE].

[23] S. Dürr, G. Koutsou and T. Lippert, Meson and baryon dispersion relations with Brillouin fermions, Phys. Rev. D 86 (2012) 114514 [arXiv:1208.6270] [INSPIRE].

[24] T. Misumi, New fermion discretizations and their applications, PoS LATTICE2012 (2012) 005 [arXiv: 1211.6999] [INSPIRE].

[25] Y.-G. Cho, S. Hashimoto, J.-I. Noaki, A. Jüttner and M. Marinkovic, $O\left(a^{2}\right)$-improved actions for heavy quarks and scaling studies on quenched lattices,

PoS LATTICE2013 (2014) 255 [arXiv: 1312.4630] [INSPIRE].

[26] Y.-G. Cho et al., Improved lattice fermion action for heavy quarks, JHEP 05 (2015) 072 [arXiv: 1504.01630] [INSPIRE].

[27] S. Dürr and G. Koutsou, On the suitability of the Brillouin action as a kernel to the overlap procedure, arXiv:1701.00726 [INSPIRE].

[28] D.H. Adams, Theoretical foundation for the index theorem on the lattice with staggered fermions, Phys. Rev. Lett. 104 (2010) 141602 [arXiv:0912.2850] [INSPIRE]. 
[29] D.H. Adams, Pairs of chiral quarks on the lattice from staggered fermions, Phys. Lett. B 699 (2011) 394 [arXiv:1008.2833] [INSPIRE].

[30] C. Hölbling, Single flavor staggered fermions, Phys. Lett. B 696 (2011) 422 [arXiv: 1009.5362] [INSPIRE].

[31] P. de Forcrand, A. Kurkela and M. Panero, Numerical properties of staggered overlap fermions, PoS LATTICE2010 (2010) 080 [arXiv:1102.1000] [INSPIRE].

[32] M. Creutz, T. Kimura and T. Misumi, Aoki phases in the lattice Gross-Neveu model with flavored mass terms, Phys. Rev. D 83 (2011) 094506 [arXiv: 1101.4239] [InSPIRE].

[33] T. Misumi, M. Creutz, T. Kimura, T.Z. Nakano and A. Ohnishi, Aoki phases in staggered-Wilson fermions, PoS LATTICE2011 (2011) 108 [arXiv:1110.1231] [INSPIRE].

[34] E. Follana, V. Azcoiti, G. Di Carlo and A. Vaquero, Spectral flow and index theorem for staggered fermions, PoS LATTICE2011 (2011) 100 [arXiv:1111.3502] [INSPIRE].

[35] P. de Forcrand, A. Kurkela and M. Panero, Numerical properties of staggered quarks with a taste-dependent mass term, JHEP 04 (2012) 142 [arXiv:1202.1867] [INSPIRE].

[36] T. Misumi, T.Z. Nakano, T. Kimura and A. Ohnishi, Strong-coupling analysis of parity phase structure in staggered-Wilson fermions, Phys. Rev. D 86 (2012) 034501 [arXiv:1205.6545] [INSPIRE].

[37] S. Dürr, Taste-split staggered actions: eigenvalues, chiralities and Symanzik improvement, Phys. Rev. D 87 (2013) 114501 [arXiv:1302.0773] [INSPIRE].

[38] C. Hölbling and C. Zielinski, Spectral properties and chiral symmetry violations of (staggered) domain wall fermions in the Schwinger model, Phys. Rev. D 94 (2016) 014501 [arXiv: 1602.08432] [INSPIRE].

[39] C. Zielinski, Theoretical and computational aspects of new lattice fermion formulations, Ph.D. thesis, Nanyang Technol. U., Singapore (2016) [arXiv:1703.06364] [INSPIRE].

[40] L.H. Karsten, Lattice fermions in Euclidean space-time, Phys. Lett. B 104 (1981) 315 [INSPIRE].

[41] F. Wilczek, On lattice fermions, Phys. Rev. Lett. 59 (1987) 2397 [INSPIRE].

[42] M. Creutz, Four-dimensional graphene and chiral fermions, JHEP 04 (2008) 017 [arXiv:0712.1201] [INSPIRE].

[43] A. Borici, Creutz fermions on an orthogonal lattice, Phys. Rev. D 78 (2008) 074504 [arXiv:0712.4401] [INSPIRE].

[44] P.F. Bedaque, M.I. Buchoff, B.C. Tiburzi and A. Walker-Loud, Broken symmetries from minimally doubled fermions, Phys. Lett. B 662 (2008) 449 [arXiv:0801.3361] [InSPIRE].

[45] P.F. Bedaque, M.I. Buchoff, B.C. Tiburzi and A. Walker-Loud, Search for fermion actions on hyperdiamond lattices, Phys. Rev. D 78 (2008) 017502 [arXiv:0804.1145] [INSPIRE].

[46] S. Capitani, J. Weber and H. Wittig, Minimally doubled fermions at one loop, Phys. Lett. B 681 (2009) 105 [arXiv:0907.2825] [INSPIRE].

[47] T. Kimura and T. Misumi, Characters of lattice fermions based on the hyperdiamond lattice, Prog. Theor. Phys. 124 (2010) 415 [arXiv:0907.1371] [INSPIRE].

[48] T. Kimura and T. Misumi, Lattice fermions based on higher-dimensional hyperdiamond lattices, Prog. Theor. Phys. 123 (2010) 63 [arXiv:0907.3774] [INSPIRE]. 
[49] M. Creutz and T. Misumi, Classification of minimally doubled fermions, Phys. Rev. D 82 (2010) 074502 [arXiv: 1007.3328] [inSPIRE].

[50] S. Capitani, M. Creutz, J. Weber and H. Wittig, Renormalization of minimally doubled fermions, JHEP 09 (2010) 027 [arXiv: 1006.2009] [INSPIRE].

[51] B.C. Tiburzi, Chiral lattice fermions, minimal doubling, and the axial anomaly, Phys. Rev. D 82 (2010) 034511 [arXiv:1006.0172] [InSPIRE].

[52] S. Kamata and H. Tanaka, Minimal doubling fermion and hermiticity, PTEP 2013 (2013) 023B05 [arXiv:1111.4536] [INSPIRE].

[53] T. Misumi, Phase structure for lattice fermions with flavored chemical potential terms, JHEP 08 (2012) 068 [arXiv:1206.0969] [INSPIRE].

[54] T. Misumi, T. Kimura and A. Ohnishi, QCD phase diagram with 2-flavor lattice fermion formulations, Phys. Rev. D 86 (2012) 094505 [arXiv: 1206.1977] [INSPIRE].

[55] S. Capitani, Reducing the number of counterterms with new minimally doubled actions, Phys. Rev. D 89 (2014) 014501 [arXiv: 1307.7497] [INSPIRE].

[56] S. Capitani, New chiral lattice actions of the Boriçi-Creutz type, Phys. Rev. D 89 (2014) 074508 [arXiv:1311.5664] [INSPIRE].

[57] T. Misumi, Fermion actions extracted from lattice super Yang-Mills theories, JHEP 12 (2013) 063 [arXiv: 1311.4365] [INSPIRE].

[58] J.H. Weber, S. Capitani and H. Wittig, Numerical studies of minimally doubled fermions, PoS LATTICE2013 (2014) 122 [arXiv:1312. 0488] [INSPIRE].

[59] J.H. Weber, Properties of minimally doubled fermions, Ph.D. thesis, Mainz U., Mainz, Germany (2015) [arXiv:1706.07104] [INSPIRE].

[60] S. Dürr and J.H. Weber, Dispersion relation and spectral range of Karsten-Wilczek and Borici-Creutz fermions, Phys. Rev. D 102 (2020) 014516 [arXiv: 2003.10803] [INSPIRE].

[61] T. Kimura, S. Komatsu, T. Misumi, T. Noumi, S. Torii and S. Aoki, Revisiting symmetries of lattice fermions via spin-flavor representation, JHEP 01 (2012) 048 [arXiv:1111.0402] [INSPIRE].

[62] A. Chowdhury, A. Harindranath, J. Maiti and S. Mondal, Many avatars of the Wilson fermion: a perturbative analysis, JHEP 02 (2013) 037 [arXiv:1301.0675] [INSPIRE].

[63] T. Misumi and J. Yumoto, Varieties and properties of central-branch Wilson fermions, Phys. Rev. D 102 (2020) 034516 [arXiv: 2005. 08857] [INSPIRE].

[64] D. West, Introduction to graph theory, Prentice Hall, Hoboken, NJ, U.S.A. (2001).

[65] J. Bondy and U. Murty, Graph theory with applications, American Elsevier Publishing Company, U.S.A. (1976).

[66] P. van Mieghem, Graph spectra for complex networks, Cambridge University Press, Cambridge, U.K. (2009).

[67] D.J. Watts and S.H. Strogatz, Collective dynamics of 'small-world' networks, Nature 393 (1998) 440.

[68] K. Ohta and N. Sakai, The volume of the quiver vortex moduli space, PTEP 2021 (2021) 033B02 [arXiv: 2009.09580] [INSPIRE]. 
[69] K. Ohta and S. Matsuura, Supersymmetric gauge theory on the graph, arXiv:2111.00676 [INSPIRE].

[70] S. Catterall, J. Laiho and J. Unmuth-Yockey, Topological fermion condensates from anomalies, JHEP 10 (2018) 013 [arXiv: 1806. 07845] [INSPIRE].

[71] N. Butt, S. Catterall, A. Pradhan and G.C. Toga, Anomalies and symmetric mass generation for Kähler-Dirac fermions, Phys. Rev. D 104 (2021) 094504 [arXiv:2101.01026] [InSPIRE].

[72] A. Kaveh and H. Rahami, A unified method for eigendecomposition of graph products, Commun. Numer. Meth. Eng. 21 (2005) 377.

[73] G. Sabidussi, Graph multiplication, Math. Z. 72 (1959) 446.

[74] F. Aurenhammer, J. Hagauer and W. Imrich, Cartesian graph factorization at logarithmic cost per edge, Comput. Complex. 2 (1992) 331.

[75] S. Noschese, L. Pasquini and L. Reichel, Tridiagonal Toeplitz matrices: properties and novel applications, Numer. Linear Alg. Appl. 20 (2012) 302.

[76] M. Gover, The eigenproblem of a tridiagonal 2-Toeplitz matrix, Linear Alg. Appl. 197-198 (1994) 63. 